\title{
Masticatory muscle pain: diagnostic considerations, pathophysiologic theories and future directions
}

\author{
Gary D. Klasser ${ }^{1}$, Jeremy Lau ${ }^{2}$, Lalima Tiwari $^{3}$, Ramesh Balasubramaniam $^{3}$ \\ ${ }^{1}$ Department of Diagnostic Sciences, School of Dentistry, Health Sciences Center, Louisiana State University, New Orleans, LA, USA; ${ }^{2}$ Perth Oral \\ Medicine and Dental Sleep Centre, West Leederville, Australia; ${ }^{3}$ UWA Dental School, The University of Western Australia, Crawley, Australia \\ Contributions: (I) Conception and design: All authors; (II) Administrative support: None; (III) Provision of study materials or patients: None; (IV) \\ Collection and assembly of data: None; (V) Data analysis and interpretation: None; (VI) Manuscript writing: All authors; (VII) Final approval of \\ manuscript: All authors. \\ Correspondence to: Gary D. Klasser. Department of Diagnostic Sciences, School of Dentistry, Health Sciences Center, Louisiana State University, New \\ Orleans, LA, USA. Email: gklass@1suhsc.edu.
}

\begin{abstract}
The importance of developing a correct and accurate diagnosis cannot be overemphasized. This principle applies to all areas of medicine and dentistry, but even more specifically to temporomandibular disorders (TMD) for which the proper diagnosis can be enigmatic and elusive. Since muscle related pain is the most common TMD diagnosis, misdiagnosis may result in the patient undergoing inappropriate invasive and irreversible interventions resulting in potential negative outcomes. This will only lead to practitioner frustration and patient dissatisfaction. Hence, an understanding of the muscle pain theories, appreciating the various diagnostic classification systems, and being knowledgeable of the various pathophysiologic theories of muscle related pain will assist the well-intentioned practitioner in avoiding the consequences of misdirected treatment. Ultimately this approach will result in prudent and properly targeted patient care. Discussion of future directions for both diagnosis and management of muscle related TMD pain will afford the reader the opportunity to enhance and objectify the diagnostic process related to masticatory muscle pain. Finally, this presentation is intended to stimulate further investigation resulting in an enrichment of our abilities to provide TMD patients with the best available evidence-based scientific management options for improvement of their condition.
\end{abstract}

Keywords: Muscle pain; diagnosis; masticatory system; temporomandibular disorders (TMD)

Received: 30 May 2020; Accepted: 22 July 2020; Published: 12 August 2020.

doi: $10.21037 /$ fomm-20-33

View this article at: http://dx.doi.org/10.21037/fomm-20-33

\section{Introduction}

For approximately $80 \%$ of patients diagnosed with temporomandibular disorders (TMD), the most common diagnosis is muscle related pain (1). In a study utilizing the Research Diagnostic Criteria for Temporomandibular Disorders (RDC-TMD) assessment criteria, the authors systematically reviewed 6 studies; $\mathrm{n}=2,491$ subjects; 1,815 women, 676 men; mean age range $23.4-46$ years, on the prevalence of masticatory muscle disorders in the general population. Despite variability among the included studies, the prevalence rates ranged from $6-13.3 \%$. A meta-analysis of the data showed an overall $9.7 \%$ prevalence (2).

In another study, utilizing a similar protocol, but focusing on patients seeking TMD treatment diagnosed with muscle disorders; $\mathrm{n}=462$; females $79.5 \%$; mean age 39.2 (range, $18-81$ ) years, there was a reported prevalence of masticatory muscle disorders of $56.4 \%$. Muscle disorders alone were diagnosed in $19.9 \%$ with a mean age of $38.6 \pm 12.9$ years.

^ORCID: 0000-0002-2033-9066. 
The authors noted that when the adoption of more rigorous diagnostic criteria and the elimination of unreliable muscle palpation sites are reappraised, the prevalence rates for TMD patients needing treatment may be more accurately reflected (3). The aim of this review is to present the various theories of muscle pain, review diagnostic classification systems, consider various pathophysiologic theories, and provide insight to the future of masticatory muscle pain diagnosis and management.

\section{Theories of muscle pain}

There are no definitive theories that totally explain why masticatory muscles become painful, the associated symptomatology, or the cause for the chronicity of muscle pain. Currently, there is no single identifiable etiologic factor, therefore muscle pain is considered "multifactorial", thereby making it more challenging to identify risk factors and their unique contributions to the process.

To date there are several hypothetical models/theories which attempt to account for muscle pain. The first was presented in 1942, the Vicious Cycle of Pain Model (VCPM) (4). It postulated that cycles of muscle hyperactivity and pain are self-perpetuating, suggesting that an initiating factor, such as abnormal posture, excessive or aberrant movement or physical stress, results in muscle pain. This theory presumes muscle pain, then leads to hyperactivity of the muscle itself or fatigue, which then perpetuates further muscle pain and dysfunction, thus continuing the cycle $(5,6)$. Unfortunately, due to potential problems associated with methodologic principles related to the characterization of muscle activity, the findings remain inconsistent. Additionally, injection of hypertonic saline (a pain producing solution) into muscle reported no changes in muscle activity $(7,8)$.

The Pain Adaptation Model (PAM) (9) proposed that alterations in muscle activity are a consequence of the presence of pain. This theory speculated that an alteration in muscle function was "adaptive," thus providing a protective mechanism for the muscle from potential damage (9). Several studies did not support the PAM (10-12). Further, PAM does not account for the psychosocial aspect of pain, neuroplasticity, genetics, and inter- or intraindividual variations in pain behavior.

The Integrated Pain Adaptation Model (IPAM), a more contemporary, broader model unifies aspects from both the VCPM and the PAM theories $(5,6)$. The IPAM hypothesized that pain affects motor activity due to its reliance on the complex interaction of distinctive biopsychosocial characteristics, as well as the anatomical and functional complexity of the individual sensory-motor system $(5,6)$. The model is based on the assumption that the masticatory system is adaptable and is capable of performing a specific task using multiple muscle recruitment pathways. However, certain aspects of this model have been unable to be reproduced in human studies $(5,6)$.

An enhancement of the IPAM, the Motor Adaptation to Pain Model (MAPM), proposes that noxious stimulation at a site results in a re-distribution of activity within and between muscles. Furthermore, incorporated into this model is the idea that changes in higher brain centers (e.g., psychosocial aspects) are an important feature in determining the ultimate nature of the redistributed motor activity (13). Despite this theory being consistent with clinical and experimental observations, it requires further validation and longitudinal studies to confirm whether the inability for adaptation is associated with long-term consequences.

\section{Diagnostic classification systems}

Developing a diagnostic classification system for any disease entity is not easy. Designing such a system specifically for TMD is an even more complicated task because both physical and psychosocial variables must be considered. According to Fillingim et al., the ideal diagnostic classification system should meet the following criteria: it should comprise all clinical diseases or disorders belonging to the field of interest, be biologically plausible so that the symptoms and signs coincide with known biological processes, there should be no overlap between disease entities because of common symptoms, clinically useful, reliable, and simple to use clinically (14).

Prior to 1992 when the RDC-TMD (15) was formulated, there was a lack of any specific universal diagnostic classification system. For most muscle pain, the majority of studies simply did not differentiate various TMD pains into specific categories making the diagnosis rather heterogenous. Therefore, analysis of the TMD literature was complicated by not knowing whether the authors were discussing muscle pain, joint pain or combined muscle and joint pain. Using a standardized and reliable examination protocol (Table 1), the RDC-TMD was the first diagnostic classification system that attempted to segregate the most common pain and non-pain-related TMD conditions. The intent of this diagnostic classification system was to 
Table 1 RDC-TMD Axis I diagnosis and Axis II psychometric instruments (15)

\begin{tabular}{ll}
\hline Axis I (physical) & Axis II (psychosocial) \\
\hline I. Muscular diagnosis & Graded Chronic Pain Scale \\
Myofascial pain & Symptom Checklist-90-Revised (only certain subscales) \\
Myofascial pain with limited opening & Functional limitation of jaw (only checklist) \\
II. DD & \\
DD with reduction & \\
DD without reduction + limited opening & \\
DD without reduction + without limited opening & \\
III. Other common joint disorders & \\
Arthralgia & \\
TMJ osteoarthritis & \\
TMJ osteoarthrosis
\end{tabular}

RDC-TMD, Research Diagnostic Criteria for Temporomandibular Disorders; TMJ, temporomandibular joint; DD, disc displacement.

include only TMD subtypes which clinical expert panels could clearly distinguish and define. Since its publication, the RDC-TMD has been translated into many languages and is the most widely employed diagnostic protocol for TMD research-based publications. It consists of a dual axis assessment by providing both physical (Axis I) and psychosocial diagnosis (Axis II) profiles. The RDC-TMD is based upon reliable and well-operationalized diagnostic criteria with the intent to simultaneously provide a physical diagnosis as well as identify other relevant patient characteristics that might influence the expression and management of their TMD.

The RDC-TMD was originally intended to be just the first step toward a universally accepted and utilized diagnostic classification system. The authors recognized that in the future there would be a need to further investigate and validate the accuracy of the Axis I diagnostic algorithms and reassess the clinical utility of Axis II instruments. Subsequently, a multicenter study to comprehensively assess the reliability and validity of the RDC-TMD was undertaken (16).

In 2014, Schiffman et al. (17) published the Diagnostic Criteria for Temporomandibular Disorders (DC-TMD), which represents an enhancement of the RDC-TMD (Table 2). The DC-TMD also employs a dual-axis assessment, which likewise incorporates both physical (Axis I) and psychosocial diagnosis (Axis II) profiles. The DC-TMD specifically separates the physical disorders into the most common pain and non-pain-related muscle and joint conditions.

One further enhancement is that DC-TMD provides a common language for both clinicians and researchers. The DC-TMD, supported by both sensitivity and specificity values for the most common muscle pain conditions, offers acceptable validity. Diagnostic algorithms for sensitivity and specificity are considered acceptable when sensitivity is $\geq 70 \%$ and specificity $\geq 95 \%$ (15). However, because the DCTMD relies solely on clinical examination, there are some limitations associated with the diagnosis of certain joint conditions. In the future, this aspect could be strengthened with the addition of temporomandibular joint (TMJ) imaging.

A supplementation and extension of the Axis I component of the DC-TMD is the expanded DC-TMD (18) (Table 3). This classification system is a mixture of 25 evidence and consensus-based masticatory muscle and TMJ conditions, an addition to the twelve most common conditions already incorporated into the existing DC-TMD. As does the previous DC-TMD, the expanded TMD taxonomy closely follows the diagnostic algorithms and clinical protocols for assessment. The diagnostic criteria for less common TMD conditions are derived from a consensus-based, expert opinion approach so field-testing is required for verification of its validity.

Other than for a preliminary diagnosis, the expanded DC-TMD deliberately excludes some extremely uncommon TMD conditions because of the paucity of data. Therefore, caution should be noted using the operationalized diagnostic 
Table 2 DC-TMD Axis I diagnosis and Axis II psychometric instruments (17)

\begin{tabular}{|c|c|c|}
\hline Axis I (physical) & Sensitivity; specificity & Axis II (psychosocial) \\
\hline Myalgia & $0.90 ; 0.99$ & Jaw Functional Limitation Scale \\
\hline Local myalgia & N/E & Patient Health Questionnaire-9 \\
\hline Myofascial pain & N/E & Patient Health Questionnaire-15 \\
\hline Arthralgia & $0.89 ; 0.98$ & Pain Drawing \\
\hline Headache attributed to TMD & $0.89 ; 0.87$ & Oral Behaviors Checklist \\
\hline \multicolumn{3}{|l|}{ Most common intra-articular TMD } \\
\hline DD with reduction & $0.34 ; 0.92^{\dagger}$ & \\
\hline DD without reduction without limited opening & $0.54 ; 0.79^{\dagger}$ & \\
\hline DJD & $0.55 ; 0.61^{\dagger}$ & \\
\hline Subluxation & $0.98 ; 0.10^{\dagger}$ & \\
\hline
\end{tabular}

${ }^{\dagger}$, Without imaging. DC-TMD, Diagnostic Criteria for Temporomandibular Disorders; TMD, temporomandibular disorders; N/E, not established; DD, disc displacement; DJD, degenerative joint disease.

criteria developed for the less common disorders listed in the expanded TMD taxonomy. This makes this expanded diagnostic classification system less than comprehensive as it is most likely to be embellished over time.

To address the need for evidence-based diagnostic criteria for the major chronic pain conditions, the Analgesic, Anesthetic, and Addiction Clinical Trial Translations, Innovations, Opportunities, and Networks (ACTTION) collaborated in a public-private partnership with the US Food and Drug Administration (FDA) and the American Pain Society (APS) to develop ACTTIONAPS Pain Taxonomy (AAPT). The long-term objective of AAPT is to advance the scientific understanding of chronic pain and its treatment (19). The AAPT has identified TMD as a common chronic pain condition, and as such has adapted the DC-TMD diagnostic criteria so that it can be translated into the AAPT framework. This framework is organized by disorder domains represented by a body system. For both research and clinical purposes each domain is comprised of five major dimensions each of which have been deemed critical in order to define, diagnose, and classify any chronic pain condition including TMD. The five domains are: diagnostic criteria common features; medical comorbidities; neurobiological, psychosocial, and functional consequences, putative neurobiological and psychosocial mechanisms; risk factors; and protective factors. Thus, within the domain of orofacial pain, the AAPT TMD was developed as an evidence-based classification system that provides a systematic incorporation of a uniformly structured set of multidimensional criteria (20) (Table 4).

Recently, a collaborative group of international individuals representing multiple organizations and associations developed the International Classification of Orofacial Pain (ICOP) (21). The organizers of this classification system felt that, to date, there was a lack of a comprehensive, internationally and universally accepted classification system that specifically addresses orofacial pains. The guiding diagnostic criteria principle introduced by the ICOP was that the characteristics of the pain disorders should be the emphasis rather than their anatomic location. The goal was to fabricate a user-friendly instrument that would enhance research and clinical management of orofacial pain. Included in ICOP are TMD diagnostic criteria which have been adopted from the DC-TMD by including only the painful TMD conditions (Table 5).

Although ICOP embraces much of the terminology and criteria presented in the DC-TMD, there are 
Table 3 Expanded taxonomy for temporomandibular disorders (supplementation and extension of the DC-TMD) (18)

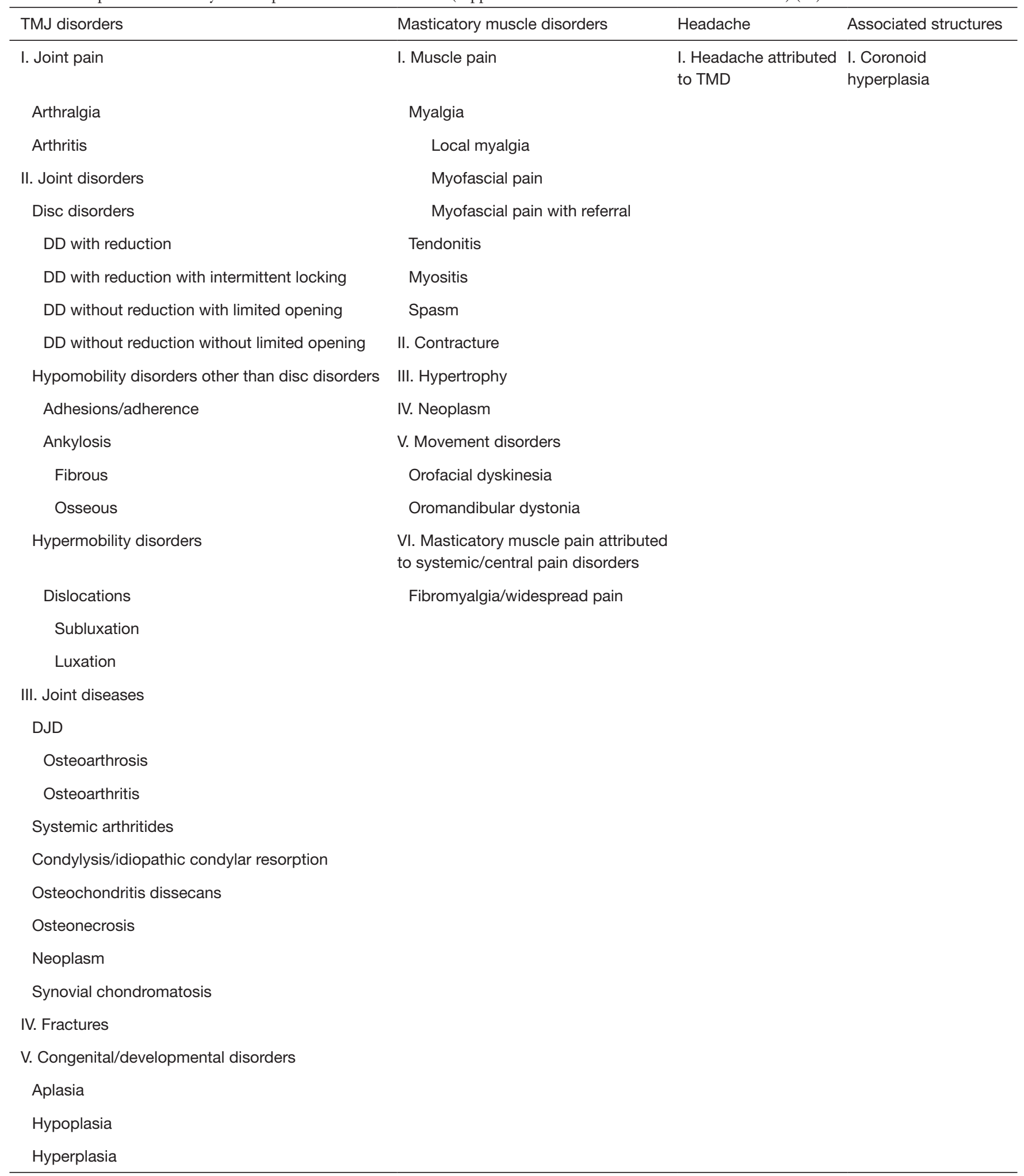

TMJ, temporomandibular joint; DC-TMD, diagnostic criteria for temporomandibular disorders; DD, disc displacement; DJD, degenerative joint disease. 
Table 4 AAPT conditions for the most common chronic painful TMD (20)

\author{
Condition \\ I. Myalgia ${ }^{\dagger}$ \\ II. Arthralgia \\ III. Headache attributed to TMD \\ IV. Painful DJD \\ V. Imaging
}

For pain to be considered chronic, within the context of the AAPT framework, the pain must have been present for $\geq 3$ months since initial onset. ${ }^{\dagger}$, Myalgia, for AAPT purposes, subsumes local myalgia, myofascial pain, and myofascial pain with referral, as defined in the DC/TMD. The implications of the subtypes of myalgia for chronicity are not currently known. ${ }^{\ddagger}$, Painful DJD represents a combination of both arthralgia and DJD, each formally defined with specific criteria and validity in the DC/TMD. The combined disorder is specific for the chronic painful TMD within the AAPT framework. AAPT, Analgesic, Anesthetic, and Addiction Clinical Trial Translations, Innovations, Opportunities, and Networks (ACTTION) and the American Pain Society (APS) Pain Taxonomy; TMD, temporomandibular disorders; DJD, degenerative joint disease.

Table 5 Painful temporomandibular conditions according to ICOP (21)

\begin{tabular}{ll}
\hline Muscle pain & Joint pain \\
\hline I. Myofascial orofacial pain & II. TMJ pain \\
Primary myofascial orofacial pain & Primary TMJ pain \\
$\begin{array}{ll}\text { Acute primary myofascial orofacial pain } \\
\text { Chronic primary myofascial orofacial pain }\end{array}$ & Acute primary TMJ pain \\
Secondary myofascial orofacial pain & Chronic primary TMJ pain \\
Myofascial orofacial pain attributed to tendonitis & Secondary TMJ pain \\
Myofascial orofacial pain attributed to myositis & TMJ pain attributed to arthritis \\
Myofascial orofacial pain attributed to muscle spasm & TMJ pain attributed to DD \\
& TMJ pain attributed to DJD \\
\hline
\end{tabular}

In primary pain conditions, the specific etiology or cause cannot be determined-that is, they are idiopathic, although substantial knowledge may exist regarding their pathophysiological mechanisms. In secondary pain conditions, the pain is secondary to, or caused by, another known medical condition or cause. According to ICOP, chronic pain is considered chronic when the onset of pain is $>3$ months. ICOP, International Classification of Orofacial Pain; TMJ, temporomandibular joint; DJD, degenerative joint disease; DD, disc displacement.

differences. Regarding muscle pain, the DC-TMD uses the terms myalgia and myofascial pain; however, ICOP employs different terminology-myofascial orofacial pain, adhering to the term myofascial in recognition of the lack of substantive evidence linking pain to specific structures or tissues located within the muscle. Additionally, ICOP incorporates a time component to diagnosis as well as distinguishing primary from secondary pain.

As new scientific information related to muscle pain is discovered, it will be necessary to expand the number of diagnostic criteria assigned to this category. Newly created muscle pain diagnostic criteria will have to be evidencebased, require cross-validation on independent samples, and be thoroughly field tested. This must apply to myofascial pain, as it is currently uncertain whether this condition can be classified as a singular disorder, or whether there are multiple subtypes. Unfortunately, no differentiated treatment algorithms exist for myalgia subtypes either. Therefore, further investigations will be required to determine whether subtypes exist, and if so, understanding their mechanisms and the clinical implications of defining these subtypes will be important (22). 


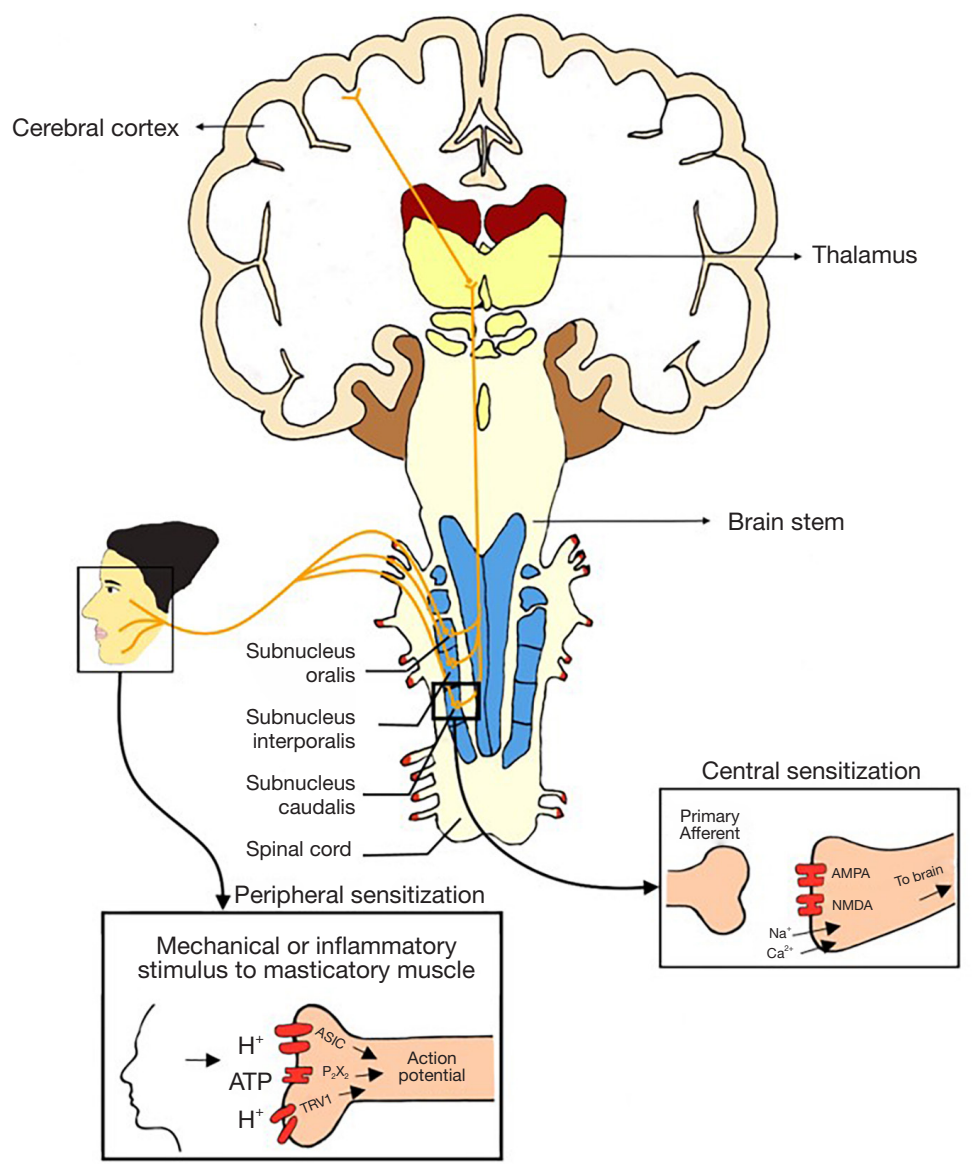

Figure 1 Pathophysiology of masticatory muscle pain: nociceptors first detect potentially harmful stimuli, before a signal is generated. Peripherally sensitization may occur leading to increased sensitivity of nociceptors. The signal then propagates towards the CNS. Overtime, central sensitization may occur at the spinal nucleus enhancing pain sensitivity. CNS, central nervous system; ATP, adenosine triphosphate.

\section{Pathophysiologic theories}

The pathophysiology of masticatory muscle pain is not well understood and remains an ongoing area of investigation. Evidence to date defines a complex multifactorial interaction at the level of the muscle, the peripheral nervous system, central nervous system (CNS) and autonomic nervous system (23).

Acute pain of the masticatory muscles results from the activation of nociceptors. Nociceptors are sensory neurons that detect potentially harmful stimuli, leading to the perception of pain, via connection to the CNS via free nerve endings $(24,25)$. These nerve endings can be sensitized and activated by strong mechanical and inflammatory stimuli, some of which have been associated with masticatory muscle pain (25). In particular, it is postulated that a decrease in $\mathrm{pH}$, the result of the activation of adenosine triphosphate (ATP) and proton $\left(\mathrm{H}^{+}\right.$ions) release can lead to activation of peripheral nociceptors and generate muscle pain. This cascade of events has been associated with several masticatory muscle pain conditions including local myalgia, myositis and myospasm (25). Furthermore, it has been demonstrated that binding of ATP to $\mathrm{P} 2 \mathrm{X}$ purinoceptor 3 ( $\mathrm{P} 2 \mathrm{X} 3$ ) molecules and $\mathrm{H}^{+}$to both transient receptor potential vanilloid 1 (TRPV1) and acid-sensing ion channels (ASICs) leads to neural excitation within masticatory muscles. This results in the generation of neuropeptides, such as substance P (SP) and calcitoningene-related peptide (CGRP), resulting in the release of endogenous inflammatory substances such as bradykinin and prostaglandin E2, contributing to the increased sensitivity of nociceptors to external stimuli, resulting in peripheral sensitization (25) (Figure 1). 
Peripheral sensitization is a recognized physiologic event whereby peripheral regions in close proximity to a painful area may themselves become painful over time, leading to an expansion of pain over more diffuse body regions. Clinical manifestations of peripheral sensitization also include the phenomena of allodynia and hyperalgesia, and may provide insight into why some muscle pain patients experience pain during normal functional muscular contractions, muscle stretching or even when the muscles are at rest (26).

Overtime, with persistence, peripheral sensitization can progress to central sensitization, further enhancing pain sensitivity. Central sensitization is an increase in the "firing" of neurons and circuits in the nociceptive pathway (27). Previously synaptic inputs that had been subthreshold or silent, now create an increased action potential output. Without central sensitization, mechanical overuse of muscles alone does not lead to the development of chronic pain (28). In patients with persistent masticatory muscle pain and myofascial pain, there is a persistence of nociceptive stimulus, coupled with central sensitization $(28,29)$.

Several other factors including psychosocial factors can become involved in this pain enhancement process. The Orofacial Pain Prospective Evaluation and Risk Assessment (OPPERA) study was the first large scale prospective study that specifically explored the role of pain amplification in TMD including hyperalgesia, allodynia and central sensitization (30). It was shown that pain amplification, similar to peripheral sensitization, leads to an increased perception of pain to a stimulus (30). Mechanisms involved in pain amplification include both decreased pain inhibitory pathways and an increase in the pain facilitatory pathways. These mechanisms have also been demonstrated in patients with TMD having lower pain threshold or reporting greater pain response to mechanical pressure or heat stimulus $(29,31,32)$. It has also been reported that individuals who are sensitive to noxious stimuli have a greater chance (risk ratio of 2.7) for developing painful TMD compared with those who are not as pain sensitive (33). Additionally, changes occur in various brain anatomic locations responsible for higher order functions, including recruitment of locations involved in eliciting emotions and subjective pain experience, as well as influencing the processes of memory and learning (34). While these studies were not specific to masticatory muscle pain, it seems reasonable to infer that the effect of pain amplification may lead to increased pain sensitivity and onset of pain in patients with masticatory muscle pain.
Autonomic variables have also been considered in playing an important role in the pathophysiology of masticatory muscle pain and TMD pain in both acute and chronic states (23,35-39). It appears that the overall autonomic balance tends to favor the sympathetic nervous system, with reduced cardiac parasympathetic tone in patients with TMD and persistent masticatory muscle pain (40). Studies indicate that TMD patients with decreased heart rate at rest displayed autonomic activity dysfunction in response to physical (orthostatic) and psychological stressors (30). Furthermore, those patients demonstrated higher heart rates when performing physical and psychological tasks, in addition to having decreased baroreceptor sensitivity (30). These findings are indicative of a central dysregulation of sympathetic activity, with resultant increased cardiosympathetic and decreased cardioparasympathetic activity, both at rest and under stress in TMD patients $(30,40)$. Furthermore, it has been theorized that the augmentation of sympathetic activity contributes to the onset and chronicity of musculoskeletal pain conditions. However, current studies lack the inclusion of important clinical, psychosocial, and genetic variables in predicting the onset of TMD and masticatory muscle pain which should be included as part of future multivariate studies investigating onset of masticatory muscle pain.

Current literature indicates that the pathophysiology of masticatory muscle pain is complex and influenced by a multitude of different risk factors. It remains unclear whether a certain risk factor leads to the onset of masticatory muscle pain, or the risk factors are the consequences of masticatory muscle pain. Another unanswered question is whether baseline pain sensitivity affects the severity and persistence of pain for those who develop masticatory muscle pain. Future investigations into masticatory muscle pain pathogenesis should look more closely at biopsychosocial risk factors, and their association with its onset and chronicity. This may provide a better understanding in identifying which patients are susceptible to chronic masticatory muscle pain.

\section{Future directions in diagnosis}

An avenue of future research which will revolutionize muscle pain diagnostics and classification emerges from the field of genetics and proteomics. Genes are intimately involved in the pain experience, however, it must be noted that pain expression relies greatly on long-term and ongoing internal and external environmental interaction which have 
the ability to alter the genes and influence the function of the proteins whose creation they initiate. Ultimately, this process has implications on how muscle pain is transmitted throughout the entire nervous system at different times throughout the entire pain experience.

Another important future consideration for muscle pain originates from neurobiologic influences involving central information processing which accounts for the central integration and processing of pain-related information travelling throughout the entire body. Important concepts to be considered in this process are those of peripheral sensitization, central sensitization, memory and learning processes and their effects on muscle pain (34). All of these biological processes will have a profound effect on the criteria assigned to various muscle pain conditions in diagnostic protocols.

Studies into the role of genetics as a risk factor for TMD has demonstrated varied results. Undoubtedly clinical and experimental pain perceptions are both influenced by genetic factors as well as environmental factors (41). Several studies have estimated the inheritability of pain related disorders associated with TMD have ranged from $34 \%$ to $58 \%(42-45)$.

To date, only a few genes have been found to be associated with TMD and masticatory muscle pain. Two particular genes that have known association in the development of TMD are the catabolic enzyme catechol-Omethyltransferase (COMT), and the intronic polymorphism of the serotonin $2 \mathrm{~A}$ receptor $(H T R 2 A)(33,4647)$. In the OPPERA study, several other genes were distinguished as potential genetic risk factors for chronic TMD including calmodulin-dependent protein kinase 4 gene (CAMK4), muscarinic cholinergic receptor 2 gene (CHRM2), G protein coupled receptor kinase 5 gene (GRK5), interferon related developmental regulator 1 gene (IFRD1) and glucocorticoid receptor gene (NR3C1) (48). In total, 358 genes are known to contribute to chronic TMD via nociceptive and inflammatory pathways. However, further research is required into their relation to masticatory muscle pain (48).

Recently, there has been research directed towards genome sequencing and epigenetics. Epigenetics can be defined as inheritance of gene expression patterns that does not involve changes in DNA sequencing. Within the field of epigenetics, the most investigated mechanism has been DNA methylation. The mechanism involves inhibition of binding of transcription factors and suppression of transcription (49). While the specific relationships associated with epigenetic and masticatory muscle pain have yet to be investigated, five gene polymorphisms have been shown to have association with TMD onset (50). Multiple PDZ domain protein $(M P D Z)$ is associated with time-based pain summation; prostaglandin-endoperoxide synthase 1 (PTGS1) and amyloid-beta precursor protein $(A A P)$ are associated with psychological symptoms and stress; while angiotensin I-converting enzyme 2 (ACE 2) and voltagegated sodium channel-type 1-alpha subunit (SCN1A) have been observed associated with nonspecific oral and facial pain symptoms (50). These key gene polymorphisms provide potential areas for further research into acute and chronic TMD onset, ultimately assisting in identifying those at risk of TMD and masticatory muscle pain at earlier stages.

Biomarkers can be utilized not only for the early diagnosis of various TMD conditions, but also to evaluate the effectiveness of treatment. There are limited studies evaluating biomarkers in the context of masticatory muscle pain. Some studies relative to local and regional muscle pain conditions have indicated that serotonin, glutamate, lactate and pyruvate were increased in patients with chronic myalgia, however further research is still required (51).

Studies have reported that dopamine neurotransmission can change centrally in chronic pain conditions, identifying it as a potential systemic biomarker (52). Typically, dopamine is increased in patients with myofascial pain. Current evidence points to the correlation of a dopamine increase with an increase in masseter muscle pain intensity (52). This suggests that dopamine is also involved in pain modulation at a peripheral level.

It has also been reported that when compared to normal controls, patients with TMD have increased levels of matrix metallopeptidases (MMPs), aggrecanase and inflammatory mediators $(53,54)$. Specifically, in patients with chronic TMD muscle pain, studies have noted an increase of amino acid secretion, elevated levels of intramuscular cytokines including IL-6, IL-7, IL-8 and IL-13 and increased salivary levels of oxidative stress biomarkers (55-57). To date, the gold standard biomarker for TMD, especially relating to masticatory muscle pain has yet to be found. Given its diagnostic potential, further research into discovering viable local and systemic biomarkers for TMD and masticatory muscle pain should be of future interest.

Brain imaging may provide another instrument that may be utilized in the future to investigate the changes in brain function and structures associated with persistent orofacial pain, especially TMD. Studies utilizing functional magnetic resonance imaging (fMRI) have provided an 
explanation for the pathological basis of neurological pain. Functional imaging provides imaging of the areas within the brain that are characterized as a "pain matrix". This matrix mainly involves the thalamus, amygdala, insular cortex, supplementary motor area, prefrontal cortex, sensory cortex and posterior parietal cortex $(58,59)$. Within the pain matrix, changes in the thalamocortical pathway of somatosensation are also evident (60). Studies have reported that with brain imaging, a common pattern of functional and structural alteration have been seen in the pain matrix of TMD patients with chronic muscle pain $(61,62)$. In addition, functional and structural changes are evident within the prefrontal cortex and limbic system in patients with chronic orofacial pain $(58,59)$. A meta-analysis highlighted functional and grey matter abnormalities in patients with chronic orofacial pain disorders particularly those involving masticatory muscles (63). Compared to healthy controls, studies have found structurally higher grey matter concentration in the posterior putamen and right ventral thalamus in patients with chronic TMD $(63,64)$. Functionally, TMD patients tended to have increased brain activity in the thalamus, but reduced activity in the insula (63). Ultimately, these brain imaging studies, although in their infancy in development, highlight the potential of fMRI as a tool for investigation and further understanding of persistent masticatory muscle pain in TMD patients.

\section{Conclusions}

The importance of an accurate diagnosis is paramount in medicine and dentistry. Without a correct diagnosis, a treatment plan has limited efficacy, and in some circumstances, may lead to treatment failure or further impairment. Since the introduction of the RDC-TMD and its evolution into DC-TMD, there is now a more standardized and reliable protocol. The diagnostic protocol continues to evolve with new research resulting one day in a standardized and validated global diagnostic protocol. The etiology and pathophysiology of masticatory muscle pain and TMD is considered multifactorial with strong ties to biologic, environmental, genetic, psychosocial, cognitive factors as well as multiple co-morbid conditions. Unfortunately, no definitive theory exists that explains the onset and chronicity associated with masticatory muscle pain. A complex interaction between the PNS and CNS is believed to contribute to the establishment of masticatory muscle pain considering the influence of peripheral and central sensitization.

fMRI research currently indicates that common patterns of functional and structural changes are reported in patients with TMD and chronic pain. The influence of genetics, epigenetics and biomarkers has the potential to be utilized for the early diagnosis and potentially early treatment or prevention of masticatory muscle pain. These and other areas of research into this field certainly portends a bright future with significant breakthroughs in patient care.

\section{Acknowledgments}

Funding: None.

\section{Footnote}

Provenance and Peer Review: This article was commissioned by the Guest Editors (Stephen Feinberg and Louis Mercuri) for the series "Temporomandibular Joint Disorders Diagnosis and Management - What Does the Future Hold?" published in Frontiers of Oral and Maxillofacial Medicine. The article was sent for external peer review organized by the Guest Editors and the editorial office.

Conflicts of Interest: All authors have completed the ICMJE uniform disclosure form (available at https://fomm. amegroups.com/article/view/10.21037/fomm-20-33/coif). The series "Temporomandibular Joint Disorders Diagnosis and Management - What Does the Future Hold?" was commissioned by the editorial office without any funding or sponsorship. The authors have no other conflicts of interest to declare.

Ethical Statement: The authors are accountable for all aspects of the work in ensuring that questions related to the accuracy or integrity of any part of the work are appropriately investigated and resolved.

Open Access Statement: This is an Open Access article distributed in accordance with the Creative Commons Attribution-NonCommercial-NoDerivs 4.0 International License (CC BY-NC-ND 4.0), which permits the noncommercial replication and distribution of the article with the strict proviso that no changes or edits are made and the original work is properly cited (including links to both the formal publication through the relevant DOI and the license). See: https://creativecommons.org/licenses/by-nc-nd/4.0/. 


\section{References}

1. List T, Dworkin SF. Comparing TMD diagnoses and clinical findings at Swedish and US TMD centers using research diagnostic criteria for temporomandibular disorders. J Orofac Pain 1996;10:240-53.

2. Manfredini D, Guarda-Nardini L, Winocur E, et al. Research diagnostic criteria for temporomandibular disorders: a systematic review of axis I epidemiologic findings. Oral Surg Oral Med Oral Pathol Oral Radiol Endod 2011;112:453-62.

3. Manfredini D, Arveda N, Guarda-Nardini L, et al. Distribution of diagnoses in a population of patients with temporomandibular disorders. Oral Surg Oral Med Oral Pathol Oral Radiol 2012;114:e35-41.

4. Travell JRS, Herman M. Pain and disability of the shoulder and arm treatment by intramuscular infiltration with procaine hydrochloride. J Am Dent Assoc 1942;120:417-22.

5. Murray GM, Peck CC. Orofacial pain and jaw muscle activity: a new model. J Orofac Pain 2007;21:263-78.

6. Peck CC, Murray GM, Gerzina TM. How does pain affect jaw muscle activity? The Integrated Pain Adaptation Model. Aust Dent J 2008;53:201-7.

7. Svensson P, De Laat A, Graven-Nielsen T, et al. Experimental jaw-muscle pain does not change heteronymous H-reflexes in the human temporalis muscle. Exp Brain Res 1998;121:311-8.

8. Matre DA, Sinkjaer T, Svensson P, et al. Experimental muscle pain increases the human stretch reflex. Pain 1998;75:331-9.

9. Lund JP, Donga R, Widmer CG, et al. The painadaptation model: a discussion of the relationship between chronic musculoskeletal pain and motor activity. Can J Physiol Pharmacol 1991;69:683-94.

10. Khawaja SN, McCall W Jr, Dunford R, et al. Infield masticatory muscle activity in subjects with pain-related temporomandibular disorders diagnoses. Orthod Craniofac Res 2015;18:137-45.

11. Kumar A, Castrillon E, Svensson KG, et al. Effects of experimental craniofacial pain on fine jaw motor control: a placebo-controlled double-blinded study. Exp Brain Res 2015;233:1745-59.

12. Ro JY, Svensson P, Capra N. Effects of experimental muscle pain on electromyographic activity of masticatory muscles in the rat. Muscle Nerve 2002;25:576-84.

13. Hodges PW, Tucker K. Moving differently in pain: a new theory to explain the adaptation to pain. Pain
2011;152:S90-8.

14. Fillingim RB, Bruehl S, Dworkin RH, et al. The ACTTION-American Pain Society Pain Taxonomy (AAPT): an evidence-based and multidimensional approach to classifying chronic pain conditions. J Pain 2014;15:241-9.

15. Dworkin SF, LeResche L. Research diagnostic criteria for temporomandibular disorders: review, criteria, examinations and specifications, critique. J Craniomandib Disord 1992;6:301-55.

16. Schiffman EL, Truelove EL, Ohrbach R, et al. The research diagnostic criteria for temporomandibular disorders. I: overview and methodology for assessment of validity. J Orofac Pain 2010;24:7-24.

17. Schiffman E, Ohrbach R, Truelove E, et al. Diagnostic criteria for temporomandibular disorders (DC/TMD) for clinical and research applications: recommendations of the International RDC/TMD Consortium Network* and Orofacial Pain Special Interest Groupdagger. J Oral Facial Pain Headache 2014;28:6-27.

18. Peck CC, Goulet JP, Lobbezoo F, et al. Expanding the taxonomy of the diagnostic criteria for temporomandibular disorders. J Oral Rehabil 2014;41:2-23.

19. Dworkin RH, Bruehl S, Fillingim RB, et al. Multidimensional Diagnostic Criteria for Chronic Pain: Introduction to the ACTTION-American Pain Society Pain Taxonomy (AAPT). J Pain 2016;17:T1-9.

20. Ohrbach R, Dworkin SF. AAPT diagnostic criteria for chronic painful temporomandibular disorders. J Pain 2019;20:1276-92.

21. International Classification of Orofacial Pain, 1st edition (ICOP). Cephalalgia 2020;40:129-221.

22. Michelotti A, Alstergren P, Goulet JP, et al. Next steps in development of the diagnostic criteria for temporomandibular disorders (DC/TMD): Recommendations from the International RDC/ TMD Consortium Network workshop. J Oral Rehabil 2016;43:453-67.

23. Svensson P, Kumar A. Assessment of risk factors for orofacial pain and recent developments in classification: implications for management. J Oral Rehabil 2016;43:977-89.

24. Gold MS, Gebhart GF. Nociceptor sensitization in pain pathogenesis. Nat Med 2010;16:1248-57.

25. Mense S. Muscle pain: mechanisms and clinical significance. Dtsch Arztebl Int 2008;105:214-9.

26. Sandkühler J. Models and mechanisms of hyperalgesia and allodynia. Physiol Rev 2009;89:707-58. 
27. Latremoliere A, Woolf CJ. Central sensitization: a generator of pain hypersensitivity by central neural plasticity. J Pain 2009;10:895-926.

28. Palla S, Farella M. Masticatory muscle pain. In: Mense S, Gerwin RD. editors. Muscle pain: diagnosis and treatment. Heidelberg: Springer, 2010:193-227.

29. Sarlani E, Greenspan JD. Evidence for generalized hyperalgesia in temporomandibular disorders patients. Pain 2003;102:221-6.

30. Maixner W, Diatchenko L, Dubner R, et al. Orofacial pain prospective evaluation and risk assessment study--the OPPERA study. J Pain 2011;12:T4-11.e1-2.

31. Maixner W, Fillingim R, Booker D, et al. Sensitivity of patients with painful temporomandibular disorders to experimentally evoked pain. Pain 1995;63:341-51.

32. Maixner W, Fillingim R, Sigurdsson A, et al. Sensitivity of patients with painful temporomandibular disorders to experimentally evoked pain: evidence for altered temporal summation of pain. Pain 1998;76:71-81.

33. Diatchenko L, Slade GD, Nackley AG, et al. Genetic basis for individual variations in pain perception and the development of a chronic pain condition. Hum Mol Genet 2005;14:135-43.

34. Dworkin SF. Research temporomandibular diagnostic criteria for disorders: current status \& future relevance. J Oral Rehabil 2010;37:734-43.

35. Slade GD, Fillingim RB, Sanders AE, et al. Summary of findings from the OPPERA prospective cohort study of incidence of first-onset temporomandibular disorder: implications and future directions. J Pain 2013;14:T116-24.

36. Perry F, Heller PH, Kamiya J, et al. Altered autonomic function in patients with arthritis or with chronic myofascial pain. Pain 1989;39:77-84.

37. Bragdon EE, Light KC, Costello NL, et al. Group differences in pain modulation: pain-free women compared to pain-free men and to women with TMD. Pain 2002;96:227-37.

38. Carlson CR, Okeson JP, Falace DA, et al. Comparison of psychologic and physiologic functioning between patients with masticatory muscle pain and matched controls. J Orofac Pain 1993;7:15-22.

39. Light KC, Bragdon EE, Grewen KM, et al. Adrenergic dysregulation and pain with and without acute beta-blockade in women with fibromyalgia and temporomandibular disorder. J Pain 2009;10:542-52.

40. Furquim BD, Flamengui LMSP, Conti PCR. TMD and chronic pain: a current view. Dental Press J Orthod 2015;20:127-33
41. Diatchenko L, Nackley AG, Tchivileva IE, et al. Genetic architecture of human pain perception. Trends Genet 2007;23:605-13.

42. Fejer R, Hartvigsen J, Kyvik KO. Heritability of neck pain: a population-based study of 33794 Danish twins. Rheumatology (Oxford) 2006;45:589-94.

43. Larsson B, Bille B, Pedersen NL. Genetic influence in headaches: a Swedish twin study. Headache 1995;35:513-9.

44. Markkula R, Järvinen P, Leino-Arjas P, et al. Clustering of symptoms associated with fibromyalgia in a Finnish Twin Cohort. Eur J Pain 2009;13:744-50.

45. Mulder EJ, Van Baal C, Gaist D, et al. Genetic and environmental influences on migraine: a twin study across six countries. Twin Res 2003;6:422-31.

46. Mutlu N, Erdal M, Herken H, et al. T102C polymorphism of the 5-HT2A receptor gene may be associated with temporomandibular dysfunction. Oral Dis 2004; 10:349-52.

47. Slade GD, Diatchenko L, Ohrbach R, et al. Orthodontic treatment, genetic factors, and risk of temporomandibular disorder. Semin Orthod 2008;14:146-56.

48. Smith SB, Maixner DW, Greenspan JD, et al. Potential genetic risk factors for chronic TMD: genetic associations from the OPPERA case control study. J Pain 2011;12:T92-101.

49. Fox SA, Tiwari L, Farah CS. Epigenetics and oral disease. In: Sonis S, Villa A. editors. Translational systems medicine and oral disease. London: Elsevier, 2020:163-206.

50. Smith SB, Mir E, Bair E, et al. Genetic variants associated with development of TMD and its intermediate phenotypes: the genetic architecture of TMD in the OPPERA prospective cohort study. J Pain 2013;14:T91101.e1-3.

51. Gerdle B, Ghafouri B, Ernberg M, et al. Chronic musculoskeletal pain: review of mechanisms and biochemical biomarkers as assessed by the microdialysis technique. J Pain Res 2014;7:313-26.

52. Dawson A, Stensson N, Ghafouri B, et al. Dopamine in plasma - a biomarker for myofascial TMD pain? J Headache Pain 2016;17:65.

53. Srinivas R, Sorsa T, Tjäderhane L, et al. Matrix metalloproteinases in mild and severe temporomandibular joint internal derangement synovial fluid. Oral Surg Oral Med Oral Pathol Oral Radiol Endod 2001;91:517-25.

54. Tominaga K, Habu M, Sukedai M, et al. IL-1 $\beta$, IL-1 receptor antagonist and soluble type II IL-1 receptor in synovial fluid of patients with temporomandibular disorders. Arch Oral Biol 2004;49:493-9. 
55. McGregor NR, Zerbes M, Niblett SH, et al. Pain intensity, illness duration, and protein catabolism in temporomandibular disorder patients with chronic muscle pain. J Orofac Pain 2003;17;112-24.

56. Louca Jounger $S$, Christidis N, Svensson P, et al. Increased levels of intramuscular cytokines in patients with jaw muscle pain. J Headache Pain 2017;18:30.

57. Rodríguez de Sotillo D, Velly AM, Hadley M, et al. Evidence of oxidative stress in temporomandibular disorders: a pilot study. J Oral Rehabil 2011;38:722-8.

58. Apkarian AV, Bushnell MC, Treede RD, et al. Human brain mechanisms of pain perception and regulation in health and disease. Eur J Pain 2005;9:463-84.

59. Apkarian AV, Hashmi JA, Baliki MN. Pain and the brain: specificity and plasticity of the brain in clinical chronic pain. Pain 2011;152:S49-64.

60. Walton KD, Dubois M, Llinás RR. Abnormal thalamocortical activity in patients with Complex Regional

doi: $10.21037 /$ fomm-20-33

Cite this article as: Klasser GD, Lau L, Tiwari L, Balasubramaniam R. Masticatory muscle pain: diagnostic considerations, pathophysiologic theories and future directions. Front Oral Maxillofac Med 2020;2:14.
Pain Syndrome (CRPS) type I. Pain 2010;150:41-51.

61. Lin CS. Brain signature of chronic orofacial pain: a systematic review and meta-analysis on neuroimaging research of trigeminal neuropathic pain and temporomandibular joint disorders. PLoS One 2014;9:e94300.

62. Scrivani S, Becerra L, DaSilva A, et al. Functional brain imaging of facial pain: functional magnetic resonance imaging (FMRI) studies of the trigeminal system. Oral Surg Oral Med Oral Pathol Oral Radiol Endod 2007;103:795.

63. Ayoub LJ, Seminowicz DA, Moayedi M. A meta-analytic study of experimental and chronic orofacial pain excluding headache disorders. Neuroimage Clin 2018;20:901-12.

64. Younger JW, Shen YF, Goddard G, et al. Chronic myofascial temporomandibular pain is associated with neural abnormalities in the trigeminal and limbic systems. Pain 2010;149:222-8. 